\title{
Detection of incoherent broadband terahertz light using antenna-coupled high-electron-mobility field-effect transistors
}

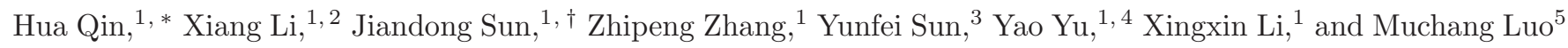 \\ 1 Key Laboratory of Nanodevices and Applications, \\ Suzhou Institute of Nano-tech and Nano-bionics (SINANO), \\ Chinese Academy of Sciences, 398 Ruoshui Road, Suzhou 215123, P. R. China \\ 2 School of Nano Technology and Nano Bionics, \\ University of Science and Technology of China, Suzhou 215123, P. R. China \\ 3 College of Electronic and Information Engineering, \\ Suzhou University of Sciences and technology, Suzhou 215009, P. R. China \\ 4 Graduate University of Chinese Academy of Sciences, Beijing 100049, P. R. China \\ ${ }^{5}$ Chongqing Institute of Optoelectronics Technology, Chongqing 400060, P. R. China
}

(Dated: March 10, 2017)

\begin{abstract}
The sensitivity of direct terahertz detectors based on self-mixing of terahertz electromagnetic wave in field-effect transistors is being improved with noise-equivalent power close to that of Schottkybarrier-diode detectors. Here we report such detectors based on AlGaN/GaN two-dimensional electron gas at $77 \mathrm{~K}$ are able to sense broadband and incoherent terahertz radiation. The measured photocurrent as a function of the gate voltage agrees well with the self-mixing model and the spectral response is mainly determined by the antenna. A Fourier-transform spectrometer equipped with detectors designed for 340, 650 and $900 \mathrm{GHz}$ bands allows for terahertz spectroscopy in a frequency range from 0.1 to $2.0 \mathrm{THz}$. The $900 \mathrm{GHz}$ detector at $77 \mathrm{~K}$ offers an optical sensitivity about $1 \mathrm{pW} / \sqrt{\mathrm{Hz}}$ being comparable to a commercial silicon bolometer at $4.2 \mathrm{~K}$. By further improving the sensitivity, room-temperature detectors would find applications in active/passive terahertz imaging and terahertz spectroscopy.
\end{abstract}

The power of nowaday terahertz imaging systems is yet greatly limited by the sensitivity of available terahertz detectors. A broadband direct detector becomes the ideal choice for passive terahertz imaging applications, while a heterodyne detector is more appropriate for active and narrow-band terahertz imaging applications [1-4]. On the other hand, terahertz Fourier-transform spectrometers (THz-FTS) become less popular in terahertz spectroscopy due to the limited detection sensitivity and dynamic range comparing to terahertz time-domain spectrometers (THz-TDS) [5, 6]. Even a FTS equipped with a liquid-helium $(4.2 \mathrm{~K})$ cooled silicon bolometer becomes less competitive with a THz-TDS [6]. Nevertheless, silicon bolometers at $4.2 \mathrm{~K}$ and pyroelectric detectors at room temperature allow for spectroscopy in a wider frequency range than a conventional THz-TDS. The main drawbacks of bolometric terahertz detectors at room temperature are the low sensitivity and the slow response speed which limit the implementation in fast imaging and spectroscopy systems. Hence, a compact, ultrasensitive and broadband terahertz detector is highly desired. Schottky-barrier diodes (SBD) based on GaAs have been well developed and are widely used in terahertz imaging systems [7]. A state-of-art SBD detector integrated in a proper waveguide offers a noise-equivalent power (NEP) about $2-12 \mathrm{pW} / \sqrt{\mathrm{Hz}}$ in a frequency range from 0.05 to $1.1 \mathrm{THz}$ [8]. When integrated with a sili-

\footnotetext{
${ }^{*}$ Corresponding author, hqin2007@sinano.ac.cn

${ }^{\dagger}$ Corresponding author, jdsun2008@sinano.ac.cn
}

con lens as a quasi-optical detector, the NEP of a SBD detector is about $10-25 \mathrm{pW} / \sqrt{\mathrm{Hz}}$ in a frequency range from 0.1 to $1.0 \mathrm{THz}$. Direct terahertz detectors based on field-effect transistors (FET) or high-electron-mobility transistors (HEMT) are merging as an alternative ultrasensitive terahertz detectors for room-temperature applications [9 19]. FET-based terahertz detectors now have sensitivity and response bandwidth both comparable to those of SBD detectors 20]. However, the wide response bandwith of FET/HEMT detectors was so far measured by using tunable single-frequency/coherent continuous-wave terahertz sources [19] or by using a train of (sub-)picosecond/broadband terahertz pulses in a THz-TDS [10, 20]. In the latter case, the Fourier components of the pulse have fixed phase relations and hence are coherent in the pulse duration. Direct detection of broadband and incoherent terahertz light from for example blackbodies by FET/HEMT detectors hasn't been demonstrated.

In this letter, we report direct detection of broadband incoherent terahertz emission from hot blackbodies by using antenna-coupled AlGaN/GaN-HEMT detectors cooled at $77 \mathrm{~K}$. The detection mechanism is examined by checking both the response spectra and the gatevoltage dependence under illumination from tunable coherent terahertz sources and broadband incoherent terahertz light. Transmission imaging of various objects illuminated by a hot wire resistor and direct imaging of hot wire resistors are demonstrated.

Detectors are designed based on the self-mixing mechanism 13 15 and fabricated using AlGaN/GaN twodimensional electron gas (2DEG) on a sapphire substrate 
(a)

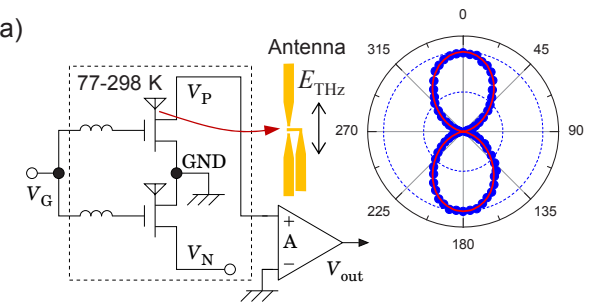

(b)

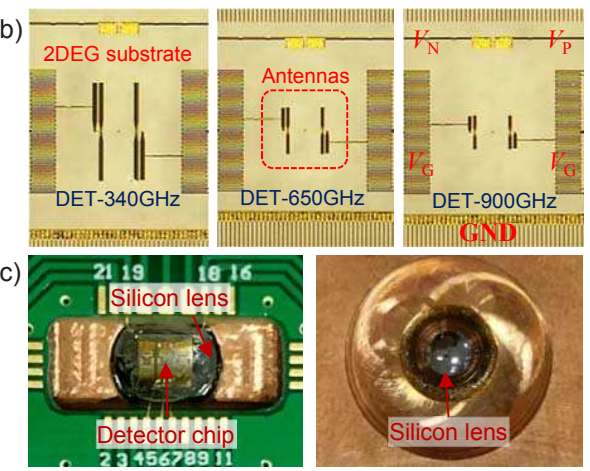

FIG. 1: (a) One of the differential antenna-coupled AlGaN/GaN-HEMTs is connected to a low-noise voltage amplifier. The inset shows the polarization characteristic of the $900 \mathrm{GHz}$ detector. (b) Partial views of detector chips designed for 340, 650 and $900 \mathrm{GHz}$ bands. (c) Backside and front-side views of the silicon hyperspherical lens with a detector chip assembled on the planar surface.

as have been reported previously [14, 16]. As shown in Fig 1(a), the detectors are configured in a differential form, i.e., two HEMTs have a common source as the ground and two differential outputs $\left(V_{\mathrm{P}}\right.$ and $\left.V_{\mathrm{N}}\right)$. The polarity of the output is determined by the asymmetric antenna. Since the antenna is of dipole style, the detector has a maximum response to terahertz wave with electric field polarized along the dipole direction, as shown in the inset of Fig. 1(a). Three detector chips named as DET-340GHz, DET-650GHz and DET$900 \mathrm{GHz}$ are fabricated with maximum response centered around $340 \mathrm{GHz}, 650 \mathrm{GHz}$ and $900 \mathrm{GHz}$, respectively, as shown in Fig. 1(b). The fabrication technique and the 2DEG properties are similar to that reported in Ref. 14. The 2DEG offers an electron density of $n_{0}=$ $0.86 \times 10^{13} \mathrm{~cm}^{-2}$ at $298 \mathrm{~K}$ and $n_{0}=1.10 \times 10^{13} \mathrm{~cm}^{-2}$ at $77 \mathrm{~K}$. Accordingly, the pinch-off voltage at which the electron density under a gate is fully depleted is $V_{\mathrm{T}} \approx-3.48 \mathrm{~V}$ at $298 \mathrm{~K}$ and $V_{\mathrm{T}} \approx-3.10 \mathrm{~V}$ at $77 \mathrm{~K}$. Electron mobility of $\mu=1,880 \mathrm{~cm}^{2} / \mathrm{Vs}$ at $298 \mathrm{~K}$ is increased to $\mu=1.54 \times 10^{4} \mathrm{~cm}^{2} / \mathrm{Vs}$ at $77 \mathrm{~K}$. The gate length is about $L=900 \mathrm{~nm}$ and the channel width is about $4 \mu \mathrm{m}$. As shown in Fig. 1(c), the detector chip is assembled in the center of the planar surface of a highresistivity silicon lens with a diameter of $6 \mathrm{~mm}$ and a height of $3.87 \mathrm{~mm}$. The detectors have a noise-equivalent power about $30-50 \mathrm{pW} / \sqrt{\mathrm{Hz}}$ at room temperature and hence doesn't show significant signal-to-noise ratio when applied to sense terahertz radiation from a blackbody with temperature below $1000 \mathrm{~K}$. However, by cooling the detectors down to $77 \mathrm{~K}$, the electron mobility is enhanced by about 8 times and the noise-equivalent power is reduced to below $10 \mathrm{pW} / \sqrt{\mathrm{Hz}}$. Hence, experiments reported in this letter were mainly conducted with the detectors cooled at $77 \mathrm{~K}$.

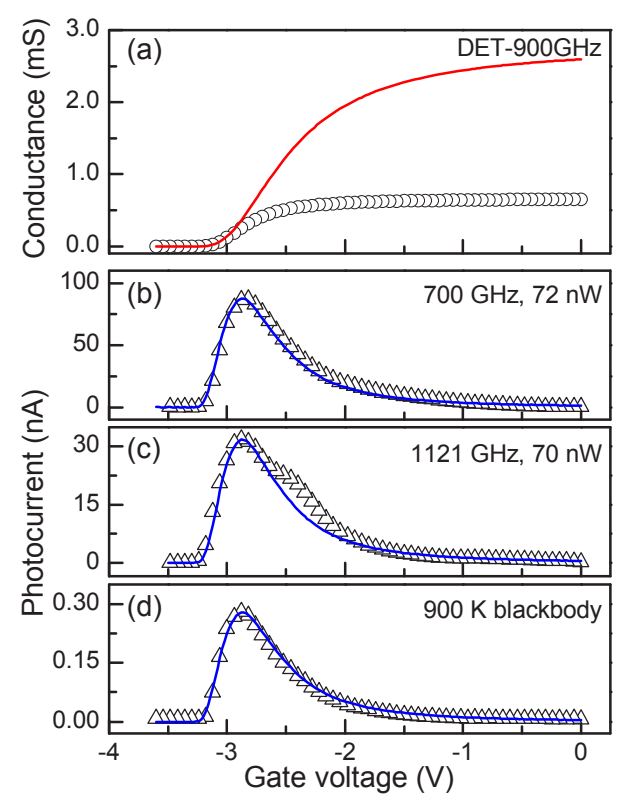

FIG. 2: (a) Total conductance $G_{\mathrm{m}}$ of DET-900GHz at $77 \mathrm{~K}$ tuned by the gate voltage. The solid curve is the gate-controlled conductance $G_{0}$ excluding the series resistance. Terahertz photocurrent tuned by the gate voltage under continuous-wave coherent irradiation at (b) $700 \mathrm{GHz}$ and (c) $1121 \mathrm{GHz}$. (d) Terahertz photocurrent induced by the incoherent broadband radiation from a blackbody with temperature about $900 \mathrm{~K}$. The solid curves in (b), (c) and (d) are the identical simulation based on the self-mixing model.

As shown in Fig. 2(a), a $900 \mathrm{GHz}$ detector gives a channel conductance about $G_{\mathrm{m}}=0.65 \mathrm{mS}$ corresponding to a total resistance of $1.54 \mathrm{k} \Omega$. The pure gatecontrolled channel conductance $G_{0}$ shown as the solid curve is extracted by excluding the large series resistance $r_{\mathrm{s}} \approx 1.15 \mathrm{k} \Omega: G_{0}=G_{\mathrm{m}} /\left(1-r_{\mathrm{s}} G_{\mathrm{m}}\right)$. The measured terahertz photocurrent $i_{\mathrm{m}}$ is only a fraction of the internal photocurrent $i_{0}$ generated in the gated channel: $i_{\mathrm{m}}=i_{0}\left(1-r_{\mathrm{s}} G_{\mathrm{m}}\right)$.

According to the self-mixing model with no sourcedrain bias applied [14], the internal photocurrent can be expressed as

$$
i_{0}=Z_{\mathrm{V}} \Xi\left(V_{\mathrm{G}}\right) M(\omega) / r_{0},
$$

where $\Xi\left(V_{\mathrm{G}}\right)=\mathrm{d} G_{0} / \mathrm{V}_{\mathrm{G}}$ is the field-effect factor, $M(\omega)$ is the self-mixing term mainly determined by the antenna design, $Z_{\mathrm{V}}=377 \Omega$ is the characteristic impedance of electromagnetic wave in vacuum and $r_{0}=1 / G_{0}$ is the channel resistance. The self-mixing term is the time average of the product of the terahertz field component 

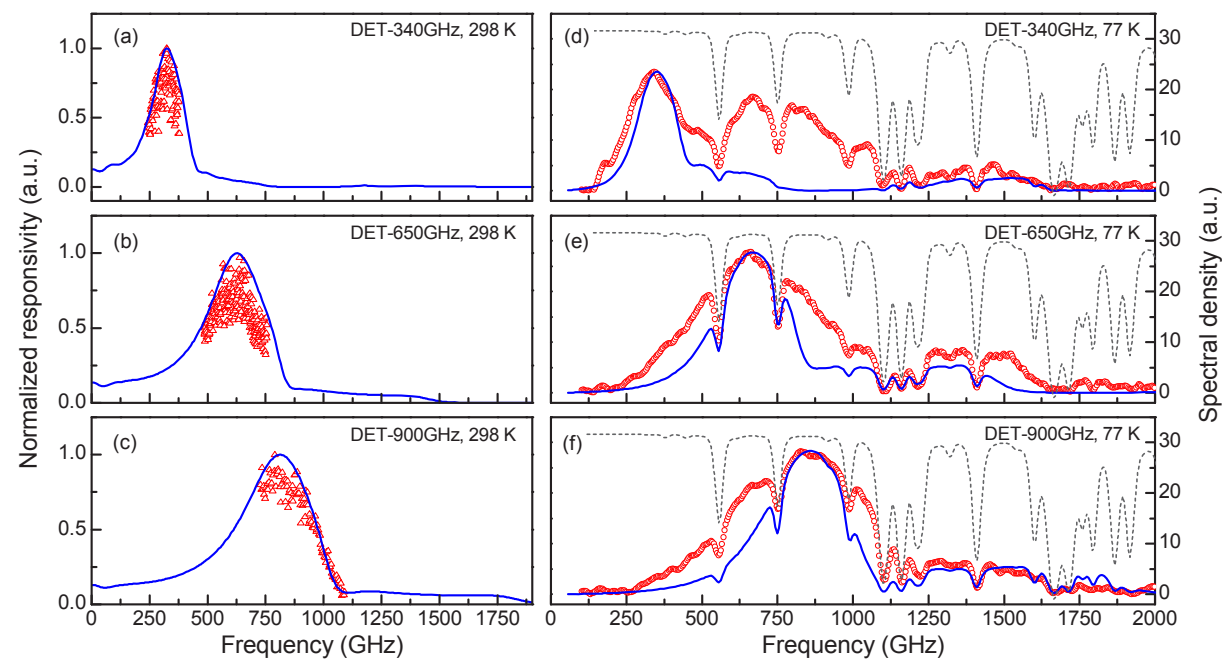

FIG. 3: (a)-(c) Normalized detector responsivity for detectors at $298 \mathrm{~K}$ by using continuously tunable coherent terahertz sources. The solid curves are the simulated self-mixing factors. (d)-(f) Measured radiation spectra of a wire resistor at $900 \mathrm{~K}$ as the incoherent broadband source by detectors at $77 \mathrm{~K}$. The solid curves are the simulated spectra and the dashed curves are the terahertz transmission coefficient in air.

along the channel and the field component perpenicular to channel

$$
M(\omega)=\left\langle\int_{0}^{L} E_{x}(x, t) E_{z}(x, t) \mathrm{d} x\right\rangle_{t} .
$$

Under irradiation of a single-frequency $(\omega)$ coherent terahertz light, the self-mixing term can be written as $M(\omega)=S_{\mathrm{A}}^{-1} \Lambda(\omega) \times I(\omega) S_{\mathrm{A}}$ where $I(\omega)=E_{0}^{2}(\omega) / 2 Z_{\mathrm{V}}$ is the Poynting flux density of the terahertz source, $S_{\mathrm{A}}$ is effective detector area, $I(\omega) S_{\mathrm{A}}$ represents the total energy sensed by the detector, $S_{\mathrm{A}}^{-1} \Lambda(\omega)$ is the self-mixing factor normalized by the incident flux density and detector area. The self-mixing factor normalized by the flux density can be expressed as

$$
\Lambda(\omega)=\int_{0}^{L} \bar{z} \dot{\xi}_{x} \dot{\xi}_{z} \cos \phi \mathrm{d} x,
$$

where $\dot{\xi}_{x}=E_{x} / E_{0}$ and $\dot{\xi}_{z}=E_{z} / E_{0}$ are the unitless field enhancement factors, $\bar{z}$ is the equivalent distance between the channel and the gate, $\phi$ is the phase difference between the in-plane field and the perpendicular field induced by the antenna. Note that phase $\phi$ is independent of time and varies from 0 at the $x=0$ to $\pi$ at $x=L$ determined by our antenna design [14].

Under irradiation from an incoherent broadband terahertz source, the induced terahertz field components seen by the channel can be expressed as $E_{x}=$ $E_{0}(\omega) \dot{\xi}_{x} \cos [\omega t+\varphi]$ and $E_{z}=E_{0}(\omega) \dot{\xi}_{z} \cos [\omega t+\varphi+\phi]$, where $\varphi$ is the random phase of each frequency component of the incoherent radiation. By substituting the incoherent electric field components into Eq. 2, the timeaveraged self-mixing factor can be expressed as

$$
M=\int_{0}^{+\infty} I(\omega) \Lambda(\omega) \mathrm{d} \omega .
$$

The form of photocurrent can be expressed by Eq. 1.

Based on the above analysis, the characteristic of terahertz photocurrent proportional to the field-effect factor and the self-mixing factor can be used as a verification of the self-mixing mechanism. As shown in Fig. 2(b) and (c), photocurrent tuned by the gate voltage is measured under continuous-wave coherent terahertz irradiation with frequency set at $700 \mathrm{GHz}$ and $1121 \mathrm{GHz}$, respectively. The maximum photocurrent occurs at an optimal gate voltage of $-2.86 \mathrm{~V}$ at which the field-effect factor is maximized $\left(\Xi_{\max }=2.44 \mathrm{mS} / \mathrm{V}\right)$. The solid curves in Fig. 2(b) and (c) are fits based on the selfmixing model and agree well with the experiment data. The extra shoulder at $-2.4 \mathrm{~V}$ in Fig. 2(c) disappears at $298 \mathrm{~K}$ and is attributed to the resonant excitation of plasma wave at $1121 \mathrm{GHz}$ as has been oberved previously [15]. Under an incoherent broadband terahertz irradiation from a blackbody with temperature about $900 \mathrm{~K}$, the photocurrent tuned by the gate voltage is plotted in Fig. 2(d). The photocurrent proportional to the field-effect factor $\Xi$ can also be well described by the same form of calculated photocurrent as shown in Fig. 2(b) and (c).

The detector spectral response to coherent continuouswave terahertz light with different frequency is examined at $298 \mathrm{~K}$ by using a continuously tunable terahertz source. In the measurement, each detector is set with the optimal gate voltage. As shown in Fig. 3(a)-(c), the normalized spectral responsivity agrees well with the corresponding simulated self-mixing factor which is determined by the antenna geometry. By cooling the detectors at $77 \mathrm{~K}$ to detect the interferogram from a terahertz Fourier-transform spectrometer, the detector spectral response to incoherent and broadband terahertz irradiation is further examined. The incoherent broadband terahertz 
light comes from a $1-\Omega$ wire resistor with a temperature about $900 \mathrm{~K}$ heated by a current. The measured blackbody spectra are shown in Fig. 3(d)-(f). Also, the product of the self-mixing factor $\Lambda(\omega)$, the blackbody spectrum $I(\omega)$ and the terahertz transmission coefficient $T_{\text {air }}(\omega)$ in air [21] is simulated for each detector and is plotted as the solid curve accordingly. The center frequencies and overall spectral shapes of the measured response spectra agree well with the simulations. The measured spectra also reveal clearly the fine absorption lines by water vapor in air. As for a reference, the terahertz transmission coefficient in air is plotted as the dashed curves. The detector has a maximum response to incoherent radiation when the polarization is along the dipole, as shown in the set of Fig. 1(a). Discrepancies between the measured and the simulated spectra are also visible. The measured spectra at $298 \mathrm{~K}$ can be fitted by the simulations much better than those obtained at $77 \mathrm{~K}$. The response spectra at $77 \mathrm{~K}$ are clearly broadened. There are a few possible causes such as a non-trivial change in the antenna impedance due to the increase in electron mobility and the rachet photocurrent from Seebeck thermoelectric effect [22, 23]. The observed photocurrent under incoherent broadband irradiation is indeed a kind of rachet effect. The antenna simulations we performed didn't take into account the 2DEG under the antenna. In the future, we would conduct more realistic simulations to examine the effect of the underlying conducting 2DEG sheet on the self-mixing factor.

Incoherent broadband terahertz emission from wire resistors with temperature about $900 \mathrm{~K}$ heated by electrical current is sensed by DET- $900 \mathrm{GHz}$ and the radiation pattern is mapped by raster scanning the resistor in the focal plane of an off-axis parabolic mirror and by fixing the detector at the focal point of the other off-axis

(a)
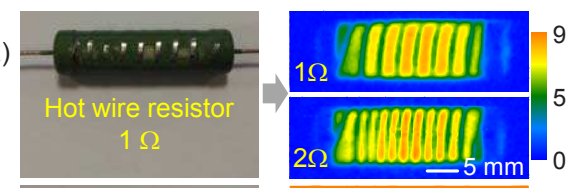

(b)

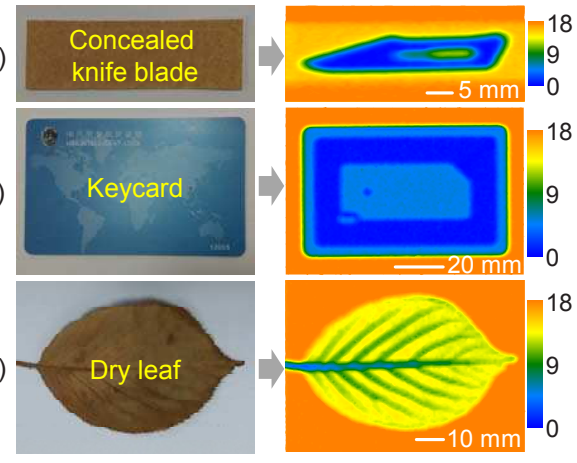

FIG. 4: (a) Hot wire resistors imaged by DET-900GHz in a raster scan. (b) A knife blade concealed in an envelope, (c) a keycard and (d) a dry leaf are imaged by DET-900GHz in raster scans under illumination from a hot resistor. parabolic mirror. As shown in Fig. 4(a), images of a $1-\Omega$ and a $2-\Omega$ wire resistors present clear temperature pattern according to the wire around the resistor body. Terahertz transmission imaging is demonstrated by using such hot wire resistor as an illumination source. As shown in Fig. 4(b), (c) and (d), a knife blade concealed in an envelope, a keycard and a dry leaf are raster scanned and the terahertz images reveal clear details of the corresponding objects.

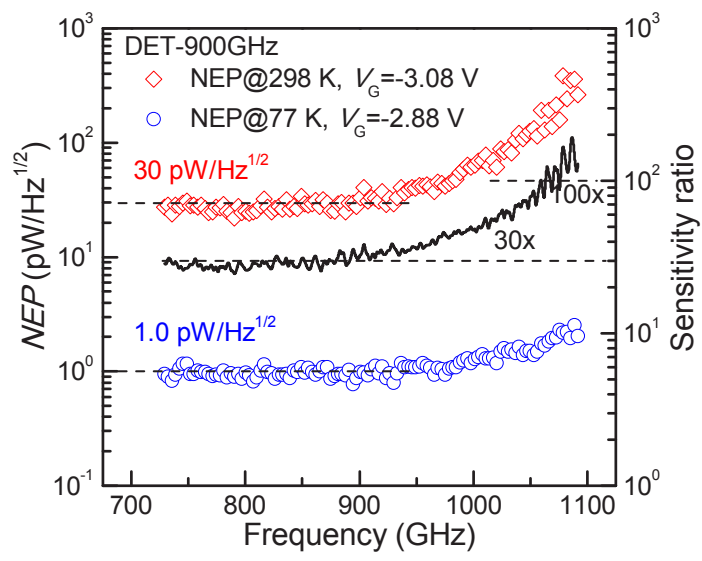

FIG. 5: Different NEPs of DET-900GHz at $298 \mathrm{~K}$ and $77 \mathrm{~K}$. The ratio of NEP at $298 \mathrm{~K}$ to that at $77 \mathrm{~K}$ is plotted to the right axis.

The optical NEP of DET-900GHz is calibrated and compared at $298 \mathrm{~K}$ and $77 \mathrm{~K}$. For calibration, a continuous-wave coherent terahertz radiation is collimated and focused into a Golay-cell detector and the total power is measured at different frequencies. The same focused terahertz light is directed to the detector and the photocurrent current is maximized by setting the optimal gate voltage. As shown in Fig. 5] an NEP of $30 \mathrm{pW} / \sqrt{\mathrm{Hz}}$ is achieved in a frequency range from $700 \mathrm{GHz}$ to $925 \mathrm{GHz}$ at $298 \mathrm{~K}$ corresponding to a bandwidth of $B \approx 200 \mathrm{GHz}$. At $77 \mathrm{~K}$, the NEP is reduced by a factor of 30 to about $1 \mathrm{pW} / \sqrt{\mathrm{Hz}}$ in the same frequency range. The detector's optical NEP becomes comparable with that of SBD detectors at room temperature and that of a commercialized silicon bolometer at $4.2 \mathrm{~K}$. The sensitivity enhancement factor by lowering the temperature can be as large as 100 with frequency above $1060 \mathrm{GHz}$. To allow for passive imaging of human bodies with a temperature sensitivity $(\Delta T)$ better than $1 \mathrm{~K}$, the detector's NEP needs to be in an order of $N E P \sim k_{\mathrm{B}} \Delta T B \sim 10^{-2} \mathrm{pW} / \sqrt{\mathrm{Hz}}$, i.e., at least three orders of magnitude to be improved for current room-temperature detectors. Recently, we have realized AlGaN/GaN-HEMT detectors with NEP below $3 \mathrm{pW} / \sqrt{\mathrm{Hz}}$ at room temperature and there are yet two orders of magnitude to be pursued.

In summary, we demonstrate detection of incoherent broadband terahertz radiation from hot blackbodies by using antenna-coupled AlGaN/GaN-HEMT detectors 
cooled at 77 K. Equipped with a Fourier-transform spectrometer, such detectors allow for terahertz spectroscopy in a wide frequency range from 0.1 to $2.0 \mathrm{THz}$. The spectral response and the responsivity tuned by the gate voltage are examined by using coherent and incoherent terahertz sources. The detector characteristics can be well described by the self-mixing model although differences are observed in response spectra when operated at $298 \mathrm{~K}$ and at $77 \mathrm{~K}$. By further improving the sensitivity, AlGaN/GaN-HEMT direct terahertz detectors would find applications in active/passive terahertz imaging and terahertz spectroscopy.

\section{Acknowledgments}

The authors acknowledge supports from the China National Natural Science Foundation (61271157, 61401456, 61401297, 61611530708), the National Key Research and Development Program of China (2016YFF0100501), the Jiangsu Science Foundation Fund (BK20140283) and the Youth Innovation Promotion Association CAS (2017372).
[1] A. Dobroiu, C. Otani, and K. Kawase, Meas. Sci. Technol. 17, R161(2006).

[2] W. L. Chan, J. Deibel, and D. M. Mittleman, Rep. Prog. Phys. 70, 1325(2007).

[3] F. Friederich, W. von Spiegel, M. Bauer, F. Meng, M. D. Thomson, S. Boppel, A. Lisauskas, B. Hils, V. Krozer, A. Keil, T. Löffler, R. Henneberger, A. K. Huhn, G. Spickermann, P. H. Bolívar, and H. G. Roskos, IEEE Trans. on Terahz. Sci. and Techn. 1, 183(2011).

[4] A. Lisauskas, M. Bauer, S. Boppel, M. Mundt, B. Khamaisi, E. Socher, R. Venckevičius, L. Minkevičius, I. Kas̆alynas, D. Seliuta, G. Valušis, V. Krozer, H. G. Roskos, J. Infrared Milli. Terahz. Waves 35, 63(2014).

[5] W. Withayachumnankul and M. Naftaly, J. Infrared Milli. Terahz. Waves 35, 610 (2014).

[6] P. Y. Han, M. Tani, M. Usami, S. Kono, R. Kersting, and X. C. Zhang, J. Appl. Phys. 89, 2357 (2001).

[7] T. W. Crowe, R. J. Matmuch, H. P. Röser, W. L. Bishop, W. C. B. Peatman, and X. L. Liu, Proc. of the IEEE, 80, 1827 (1992).

[8] http://www.vadiodes.com/index.php/en/products-6/detectors

[9] M. I. Dyakonov and M. S. Shur, IEEE Trans. Electron Devices 43, 380(1996).

[10] F. Teppe, D. Veksler, V. Yu. Kachorovski, A. P. Dmitriev, X. Xie X.-C. Zhang, W. Knap and M. S. Shur Appl. Phys. Lett. 87, 022102 (2005).

[11] E. Öjefors, A. Lisauskas, D. Glaab, H. G. Roskos, U. R. Pfeiffer, J. Infrared Milli. Terahz. Waves 30, 1269 (2009).

[12] F. Schuster, D. Coquillat, H. Videlier, M. Sakowicz, F. Teppe, L. Dussopt, B. Giffard, T. Skotnicki, and W. Knap, Optics Express 19, 7827(2011).

[13] Y. F. Sun, J. D. Sun, Y. Zhou, R. B. Tan, C. H. Zeng, W. Xue, H. Qin, B. S. Zhang, and D. M. Wu, Appl. Phys. Lett. 98, 252103 (2011).
[14] J. D. Sun, H. Qin, R. A. Lewis, Y. F. Sun, X. Y. Zhang, Y. Cai, D. M. Wu, and B. S. Zhang, Appl. Phys. Lett. 100, 173513 (2012).

[15] J. D. Sun, H. Qin, R. A. Lewis, X. X. Yang, Y. F. Sun, Z. P. Zhang, X. X. Li, X. Y. Zhang, Y. Cai, D. M. Wu, and B. S. Zhang, Appl. Phys. Lett. 106, 031119 (2015).

[16] J. D. Sun, Y. F. Sun, D. M. Wu, Y. Cai, H. Qin, and B. S. Zhang, Appl. Phys. Lett. 100, 013506 (2012).

[17] W. Knap, S. Rumyantsev, M. S. Vitiello, D. Coquillat, S. Blin, N. Dyakonova, M. Shur, F. Teppe, A. Tredicucci, and T. Nagatsuma, Nanotech. 24, 214002 (2013).

[18] T. Watanabe, S. A. Boubanga-Tombet, Y. Tanimoto, D. Fateev, V. Popov, D. Coquillat, W. Knap, Y. M. Meziani, Y. Wang, H. Minamide, H. Ito, and T. Otsuji, IEEE Sensors Journal 13, 89 (2013).

[19] M. Bauer, R. Venckevičius, I. Kas̆alynas, S. Boppel, M. Mundt, L. Minkevičius, A. Lisauskas, G. Valušis, V. Krozer, and H. G. Roskos, Optics Express 22, 19250(2014).

[20] S. Preu, S. Regensburger, S. Kim, M. Mittendorff, S. Winnerl, S. Malzer, H. Lu, P. G. Burke, A. C. Gossard, H.B. Weber, and M.S. Sherwin, Proc. of SPIE 8900, 89000R (2013).

[21] The AM atmospheric model. Smithsonian Astrophysical Observatory, http://www.fa.harvard.edu/ spaine/am

[22] V. V. Popov, D. V. Fateev, E. L. Ivchenko, and S. D. Ganichev, Phys. Rev. B 91, 235436 (2015).

[23] P. Olbrich, J. Kamann, M. König, J. Munzert, L. Tutsch, J. Eroms, D. Weiss, Ming-Hao Liu, L. E. Golub, E. L. Ivchenko, V. V. Popov, D. V. Fateev, K. V. Mashinsky, F. Fromm, Th. Seyller, and S. D. Ganichev, Phys. Rev. B 93, 075422 (2016). 